\title{
Adapting menus in care homes to meet The Foods Standards Agency guidelines: a qualitative study of barriers and facilitators to change
}

\author{
B. Heaven ${ }^{1}$, C. Bamford ${ }^{1}$, C. May ${ }^{1}$ and P. Moynihan ${ }^{2,3}$ \\ ${ }^{1}$ Institute of Health and Society, ${ }^{2}$ Institute for Ageing and Health and ${ }^{3}$ Human Nutrition Research Centre, \\ Newcastle University, Newcastle upon Tyne, NE2 4BW, UK
}

The Food Standards Agency (FSA) has developed guidelines for the nutrition of older people living in residential care ${ }^{(1)}$. As part of a broader study investigating the impact of implementing the FSA guidelines on the menus, resident's food consumption and food waste, a qualitative investigation to identify the barriers and facilitators to implementing the guidelines was conducted.

Ethnographic methods, including semi-structured interviews, informal focus groups and direct observations were used in five care homes in the North of England to explore the practices, preferences and views of residents, catering, care and senior staff and the views of relatives. Data were collected at baseline, and at 1 and 6 months after changes to the menu. In total, 79 care home staff, 29 clients and 3 relatives were interviewed across all five homes and time points. Observation periods lasted between half an hour to $5 \mathrm{~h}$ in a single session, and were written up in field notes. The field notes and transcripts of audio recorded interviews constituted the data for analysis. Analysis occurred using the constant comparative method ${ }^{(2)}$, and was informed by Normalisation Process Theory ${ }^{(3)}$.

A wide range of facilitators and barriers to the adaptation of existing menus within each home was found although some themes were common to all homes. Ubiquitous themes included the importance of encouraging 'ownership' in the adapted menus for both kitchen and care staff, ensuring that adapted menus were flexible and fitted the local context of the home, and allowing enough time and support for the practicalities of change. A key barrier to adapting the menus was a perception among some staff and residents that the FSA guidelines were a form of reducing diet, or means of deprivation. Some staff questioned the principal of the FSA guidelines for older people living in residential care. A common response was that there was no point in changing the diet of the residents, as they might not live long enough to experience potential benefits. However, training was adapted to point out the short-term benefits to older people of adapting diet, and some staff responded positively to this intervention.

The FSA guidelines were not accepted in their entirety in any of the five homes. The process of adapting the menu was most successful in homes that were both 'ready for change,' and where the concept of 'eating for wellbeing' was seen as important by the staff, and applicable for the residents. Readiness for change was observed in three homes where the existing menu was being considered for revision prior to the study. In addition, in one home a new cook had been employed, and the process of adapting the menu coincided with her efforts to establish a fresh approach. Successful and sustained change depended on the availability and practical input of a research Dietitian to support appropriate adaptations to the menus. In particular, all cooks across the five homes, regardless of nutritional knowledge or training, required the input of a Dietitian to successfully adapt their menus towards the FSA guidelines. A robust training programme for care home cooks in nutritional knowledge is therefore recommended, although this may not be sufficient for adapting menus without additional dietetic support. It is concluded that in order to adapt care home menus towards the FSA guidelines, a comprehensive support strategy is required for all staff including financial, organisational and practical (dietetic) assistance and training.

This research was commissioned by the Food Standards Agency, project N14010. The views expressed are those of the authors.

1. Food Standard Agency (2007) Nutrient and food based guidelines for UK institutions. http://www.food.gov.uk/multimedia/pdfs/nutguideuk.pdf

2. Glaser B \& Strauss A (1967) The Discovery of Grounded Theory. Chicago: Aldine

3. May C (2006) A rational model for assessing and evaluating complex interventions in health care. BMC Health Serv Res 6. 